\title{
Primary malignant melanoma of the orbit arising in a cellular blue naevus*
}

\author{
K U LÖFFLER AND H WITSCHEL \\ From the Department of Ophthalmology, Freiburg University, FRG
}

SUMMARY Primary malignant melanoma of the orbit is a rare clinical entity. We report the clinical course and present the light and electron microscopic findings in a case arising in a cellular blue naevus. While most orbital melanomas are associated with a naevus of Ota, there was no clinically visible abnormal pigmentation in our patient. The possible origin of the tumour cell and its ultrastructural features are discussed.

Primary orbital melanomas are extremely rare. Reports are few,,$^{1-8}$ and in most cases the tumours are associated with oculodermal melanosis (naevus of Ota). We present a case of a very slowly progressing malignant melanoma of the orbit without clinically visible pigmentation. It was a diagnostic problem as well as a problem in terms of treatment and management of the patient. We also discuss our ultrastructural findings with regard to the origin of the tumour cell and the specific features associated with a certain localisation of a melanocyte. ${ }^{9}$

\section{Case report}

In May 1980 a 27-year-old male presented at our clinic with slight proptosis of his right eye. No other pathological findings could be detected. When reexamined in December 1980 a right orbital mass was present, indenting the globe inferiorly and nasally. CT scan showed a solid, non-fatty, and moderately vascularised lesion. Because of recently developed pain a biopsy was performed two months later. Histologically this was reported to be a blue naevus whereas the clinical impression was that of a varix. By September 1982 all symptoms had vanished.

In December 1984 the patient complained again of pain and exophthalmus, so another biopsy was done and a 'cyst' was drained. Several experts were consulted on the histology of the specimen. There

*Presented in part at the Annual Meeting of the German Ophthalmic Pathologists, Heidelberg, September 1987.

Correspondence to Dr Karin U Löffler, Universitäts-Augenklinik, Killianstr.5, 7800 Freiburg, FRG. was, however, no uniform opinion on the diagnosis and malignancy of the lesion, opinion ranging from lymphatic tumour to naevus and to malignant melanoma. Clinically it still appeared as a varix. In the meantime the patient had again recovered completely. It was not until February 1987 that severe pain necessitated another biopsy (histology no. 9785). The final diagnosis was now made and the right orbit was exenterated (histology no. 9801). Visual acuity had always remained better than $5 / 6$.

\section{PATHOLOGICAL FINDINGS}

\section{Biopsy specimen 9785}

On macroscopic examination the specimen consisted of several fragments of soft blood-stained tissue taken from the 'capsule' as well as from the central part of the tumour. They were fixed separately in $2 \%$ glutaraldehyde, and a fragment from the capsule was also fixed in formalin. Some tisue fixed in glutaraldehyde was kept for electron microscopy; the remaining fragments were processed for routine light microscopy. Macroscopically there were no specific features.

On light microscopy the specimen taken from the tumour periphery showed a collagenous matrix containing several nests of naevus cells (Fig. 1), some of which were heavily pigmented, whereas others were pale and of rather neuroid appearance. Adjacent to this tissue the histological features were similar to those of the material which was removed from the central part of the tumour. Here the cells were tightly packed without deposition of any extracellular sub- 


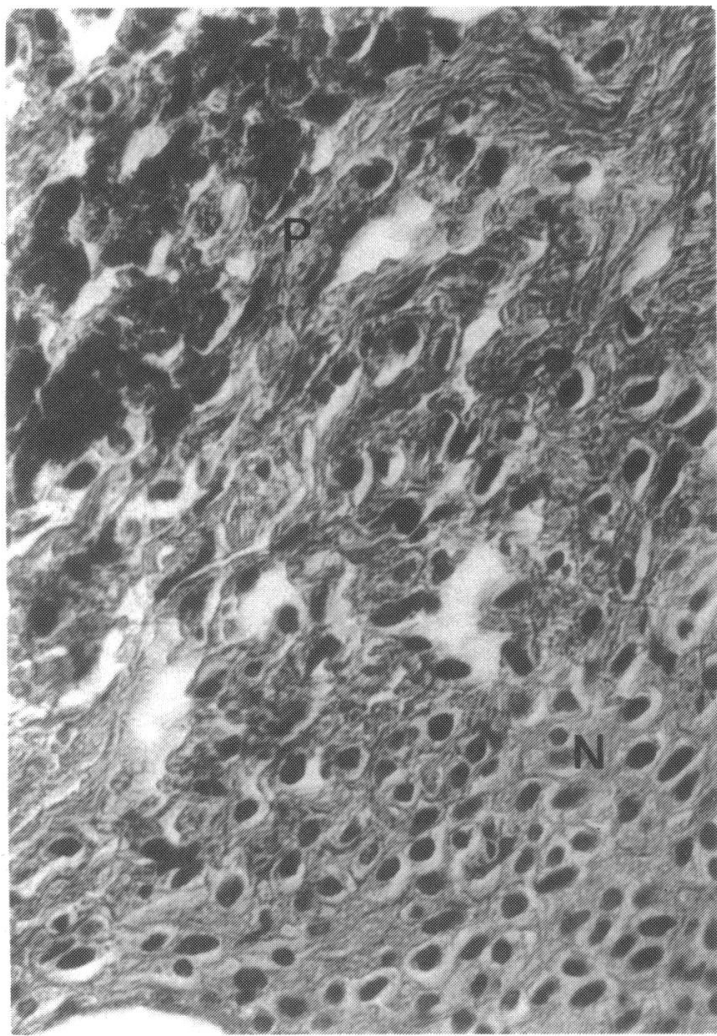

Fig. 1 Biopsy (9785) taken from the tumour periphery, showing features of a cellular blue naevus. Note heavily pigmented cells $(\mathrm{P})$ and lighter cells of neuroid appearance (N). Haematoxylin-eosin, $\times 730$.

stances (Fig 2). They were of spindle to epithelioid cell type with prominent nucleoli, and mitotic figures could be seen in a varying degree. There was some cellular pleomorphism and nuclear atypia as well as haemorrhage and necrosis in some areas. Melanin could be detected only with difficulty, even when using the Masson-Fontana stain.

For electron microscopy small blocks of the 'capsule' (7-87) and of tumour tissue (8-87) were dehydrated through graded alcohol and embedded in Araldite. Semithin sections were cut with a ReichertJung Ultracut microtome and stained with toluidine blue. Ultrathin sections were cut with the same microtome, stained with uranyl acetate and lead citrate, and visualised with a Zeiss 109 electron microscope.

The light microscopic impression of two populations of naevus cells could be confirmed ultrastructurally (Fig. 3). Embedded in bundles of collagen fibres, one cell type showed dark nuclei with clumped chromatin but no distinct nucleolus and contained

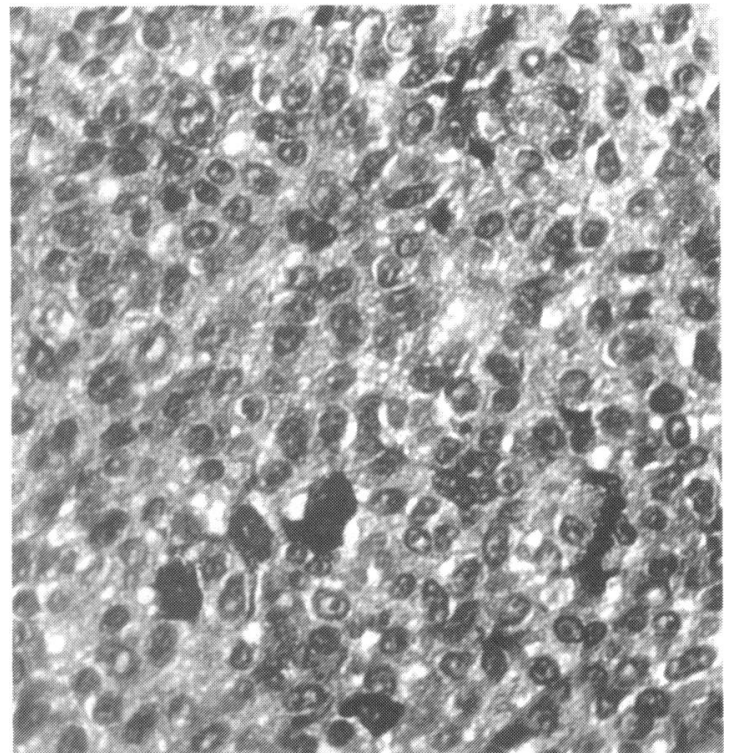

Fig. 2 Biopsy (9785) taken from the central part of the tumour, showing tightly packed cells with prominent nucleoli. Only a few cells contain pigment, and no extracellular matrix can be identified. Haematoxylin-eosin, $\times 730$.

numerous melanosomes of different stages and size. The other cell type had a more electron-lucent nucleus and fewer cytoplasmic organelles. Several autophagolysosomal collections of melanin, which are only rarely seen in ordinary naevus cells, were rather conspicuous, and occasionally a basement membrane could be observed.

The melanoma consisted of polyhedral cells lying close together (Fig. 4). Even by electron microscopy no extracellular matrix formation could be seen. Most of the cellular nuclei were deeply indented or showed nuclear pockets; the chromatin was finely dispersed without margination at the nuclear membrane, and large wiry nucleoli were a prominent feature.

The cytoplasm contained numerous mitochondria and endoplasmic reticulum as well as golgi zones and sometimes basal bodies were easily visible (Fig. 5). The rough endoplasmic reticulum often showed a conspicuous whorl formation. In most tumour cells the cytoplasm was of a granular substructure due to free ribosomes, but in some cells cytoplasmic filaments were present (Fig. 6). Premelanosomes were not seen in the sections examined, and hardly any melanin granules or melanophagosomes could be identified (Fig. 5). Occasionally melanosome-like bodies were seen next to a Golgi apparatus. These cells, however, also contained neurosecretory 


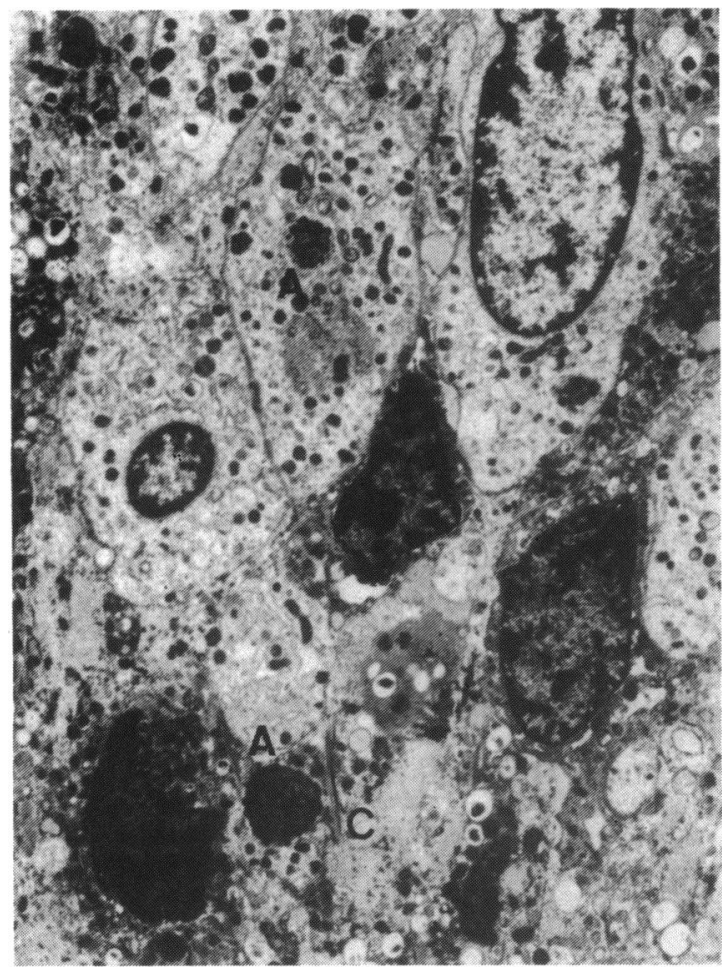

Fig. 3 Ultrastructural appearance of naevus cells (7-87). Note difference in nuclear chromatin and cytoplasmic density. A: autophagolysosomal accumulation of melanin. C: collagen fibres. $\times 4700$ ).

granules and appeared to be of Schwann cell origin (Fig. 7), though a basement membrane could not be demonstrated.

The individual cell configuration often showed prominent interdigitations and rudimentary elongated cellular processes indicative of abortive dendrite formation. Many desmosome-like junctions were present (Figs. 5, 6), and in addition we found one rather unusual form of cellular connection which might be the ultrastructural appearance of a very late mitotic figure: the cell membrane of two adjacent cells was interrupted for a distance of $0 \cdot 3 \mu \mathrm{m}$, and this gap was traversed by a bundle of intermediate filaments approximately $10 \mathrm{~nm}$ in diameter (Fig. 8).

\section{Exenteration specimen 9801}

On macroscopic examination the specimen measured $50 \times 46 \times 40 \mathrm{~mm}$ with a nodular mass in the posterior inferonasal quadrant (approximately $26 \times 23 \times 18$ $\mathrm{mm}$ ). After fixation in $2 \%$ glutaraldehyde an oblique section was taken across the globe and the tumour. The tumour cut surface showed lobulated whitish tissue with only slight pigment dusting at the centre

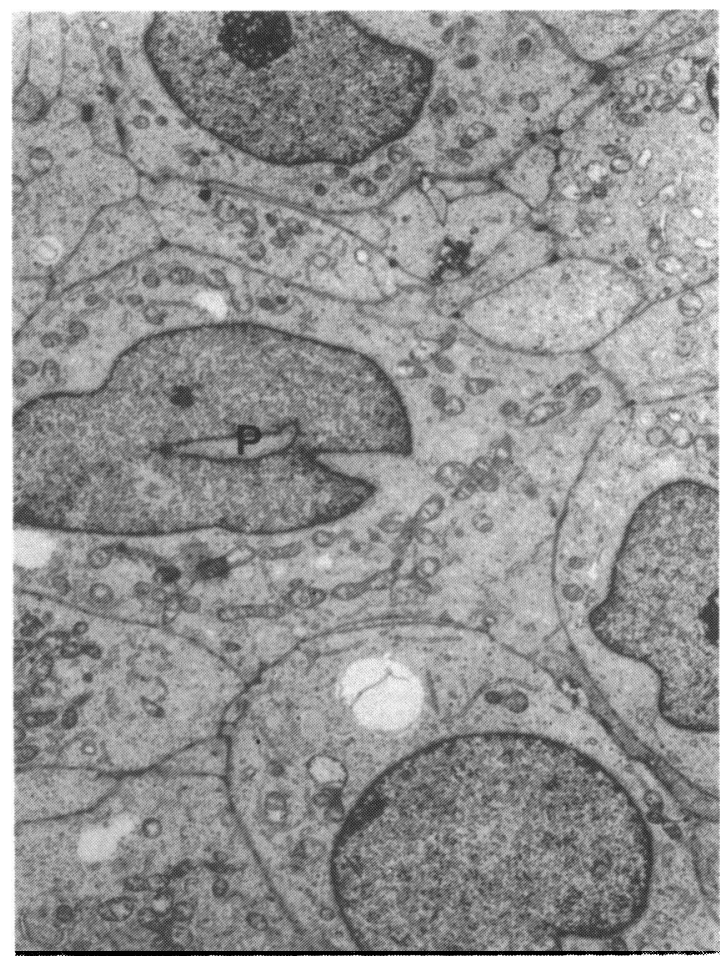

Fig. 4 Ultrastructural appearance of melanoma cells (8-87). Note prominent nucleoli and nuclear pocket $(\mathrm{P})$ and abundant mitochondria. $\times 4700$.

and some foci of heavier pigmentation at the periphery. There were also areas of necrosis, and on the second cut surface there was scar tissue indicating the site of the previous biopsy. No definite capsule was seen. The eye itself appeared unremarkable; in particular there was no evidence of any intraocular tumour. No particular pigmentation within the globe was noted.

The light microscopic appearance of the tumour was similar to that seen in the biopsy specimen. At the periphery there were heavily pigmented naevus cells embedded in a collagenous matrix, whereas the main tumour mass consisted of rather tightly packed cells of spindle or epithelioid type with prominent nucleoli. Even when using the Masson-Fontana stain for melanin hardly any pigmentation could be detected, and mitotic figures were inconspicuous. Only small foci of lymphocytic infiltration were seen. There were, however, large areas of tumour necrosis and degeneration. At the site of the previous biopsies there was some reactionary scar tissue with deposition of iron pigment. No significant pathological changes could be detected in the ocular tissues: there was only marked peripheral microcystoid degenera- 


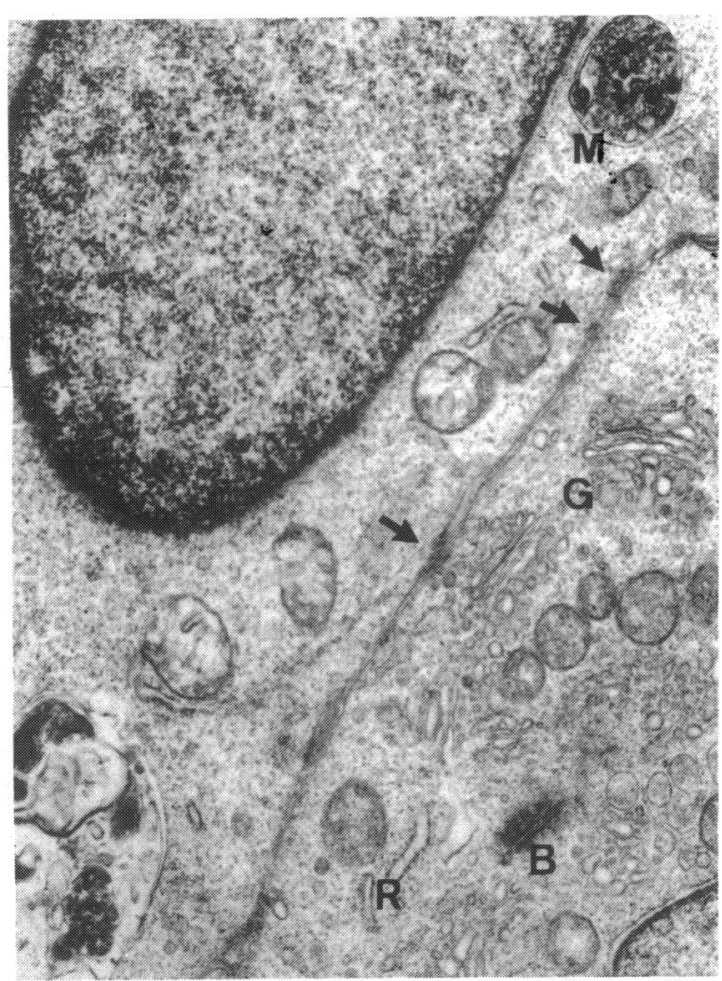

Fig. 5 Tumour cell with prominent golgi apparatus $(\mathrm{G})$, endoplasmic reticulum (R), and basal body (B). Note melanophagosome $(\mathrm{M})$ in adjacent cell and several desmosome-like cell junctions (arrows). $\times 17600$.

tion of the retina and an artificial detachment of the choroid in some parts. There was no evidence of uveal hyperpigmentation or intraocular tumour formation, nor was there any indication of scleral invasion by the orbital melanoma.

Owing to inadequate fixation for electron microscopy the quality of the ultrathin sections was rather poor. We therefore concentrate on our ultrastructural findings in the biopsy specimen. Basically, the main morphological features were-as already shown by light microscopy-similar in both specimens.

\section{Discussion}

Only few cases of primary malignant melanoma of the orbit have been reported, ${ }^{1-8}$ and until now there has not been much information on their clinical behaviour.

Quite often primary orbital melanoma is associated with a naevus of Ota, showing a heavily pigmented skin and/or choroid at the site of the involved orbit. ${ }^{2358}$ In our patient, however, there

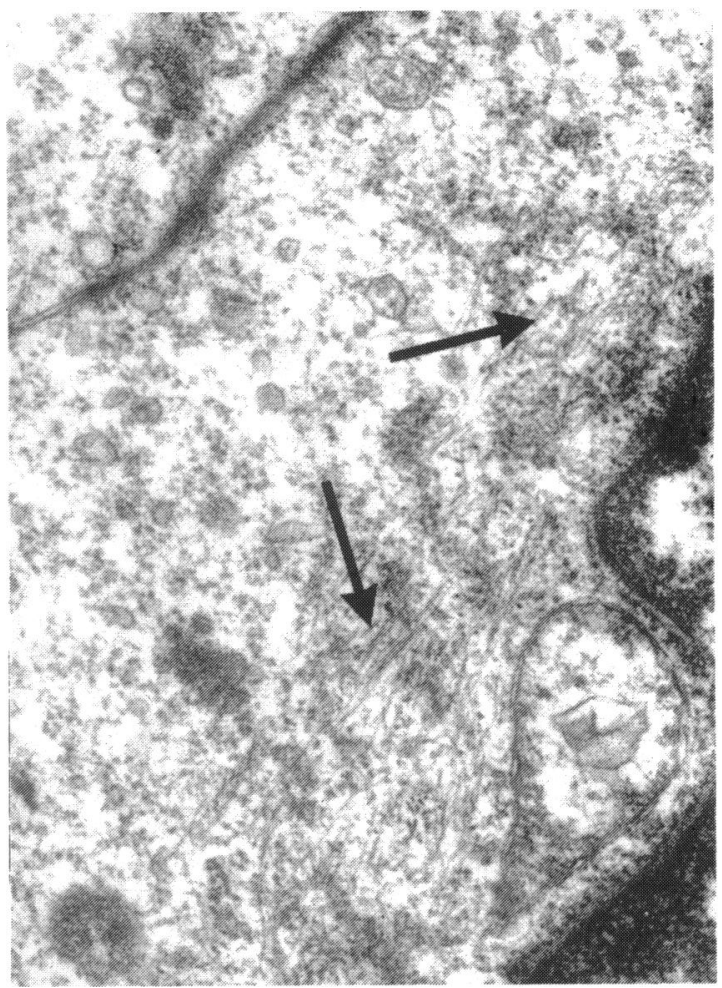

Fig. 6 Tumour cell with cytoplasmic filaments (arrows). Again, many intercellular junctions are present. $\times 33000$.

was no visible sign of any abnormal pigmentation suggestive of a melanocytic lesion. In addition the clinical (that is intraoperative) impression was much more in favour of a vascular process, since the tissue removed was rather soft, being 'encapsulated' by some firmer tissue. The first biopsy histologically excluded the suspected varix but was reported as showing a benign naevus. This diagnosis was supported by the long-standing history and the lack of any severe symptoms until a further biopsy had to be taken because of painful proptosis seven years after the onset of the disease. By that time there was definite evidence of malignant transformation. It therefore seems of great importance to consider the possibility of malignancy despite a benign clinical course. This is supported by some reports describing orbital melanomas which developed and expanded rather slowly. ${ }^{147}$

The cellular origin of the orbital melanoma is not clear. Although in our case there was a cellular blue naevus as a predisposing lesion, this only transfers the problem to the origin of the naevus cell. It has been suggested that melanocytes of the optic nerve sheath can proliferate and undergo malignant trans- 


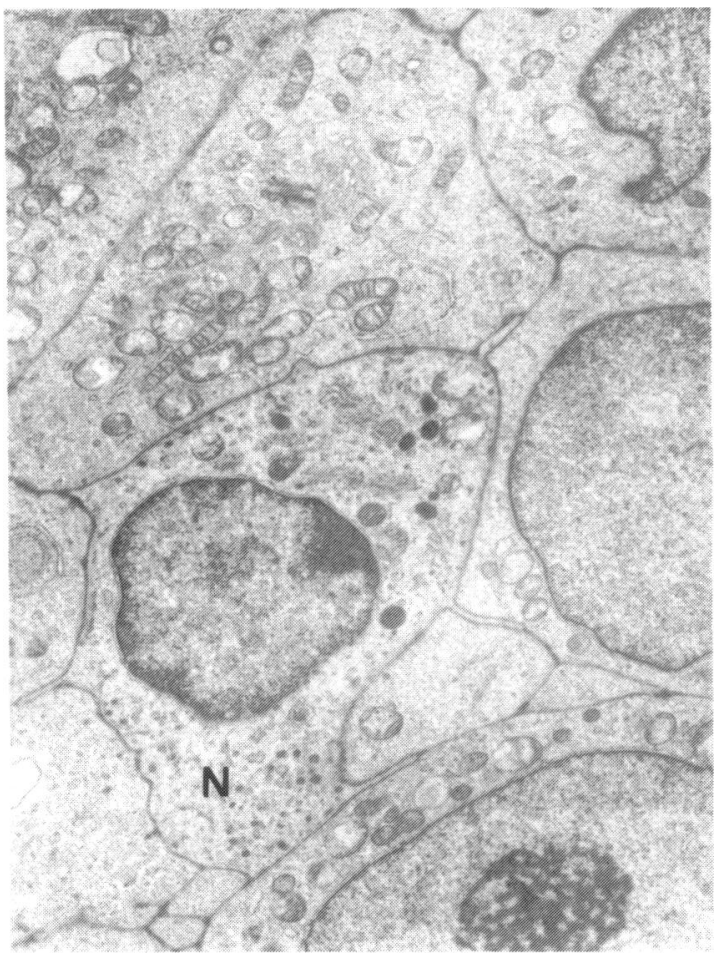

Fig. 7 Cell within melanoma tissue, containing neurosecretory granules $(\mathrm{N}) . \times 7600$.

formation. This seems unlikely in our patient, since the tumour and in particular the naevus part did not show any connection with the optic nerve and was located in the inferonasal quadrant of the orbit. Indeed there is some evidence of Schwann cell involvement (Fig. 7), which might indicate either a relationship to a peripheral nerve as seen in desmoplastic (neurotropic) melanoma ${ }^{1011}$ or confirm the theory that neoplastic cells in a cellular blue naevus are of Schwann cell origin. ${ }^{12}$

As to the malignant behaviour of a cellular blue naevus, this is well established. ${ }^{12}{ }^{13}$ Presumably there is a similar aetiology for the cellular blue naevus in our patient as for a naevus of Ota - that is, we are dealing with cells which have persisted during development and did not regress as in 'normal' individuals. Thus those hamartomatous cells not responding to growth suppressive factors might already have some genetic defect which makes them more likely to transform into uncontrolled true malignant cells.

On ultrastructural grounds we could not find any particular features specific for 'orbital' malignant melanoma. Since there were so few melanosomes, we do not feel justified in drawing any conclusions

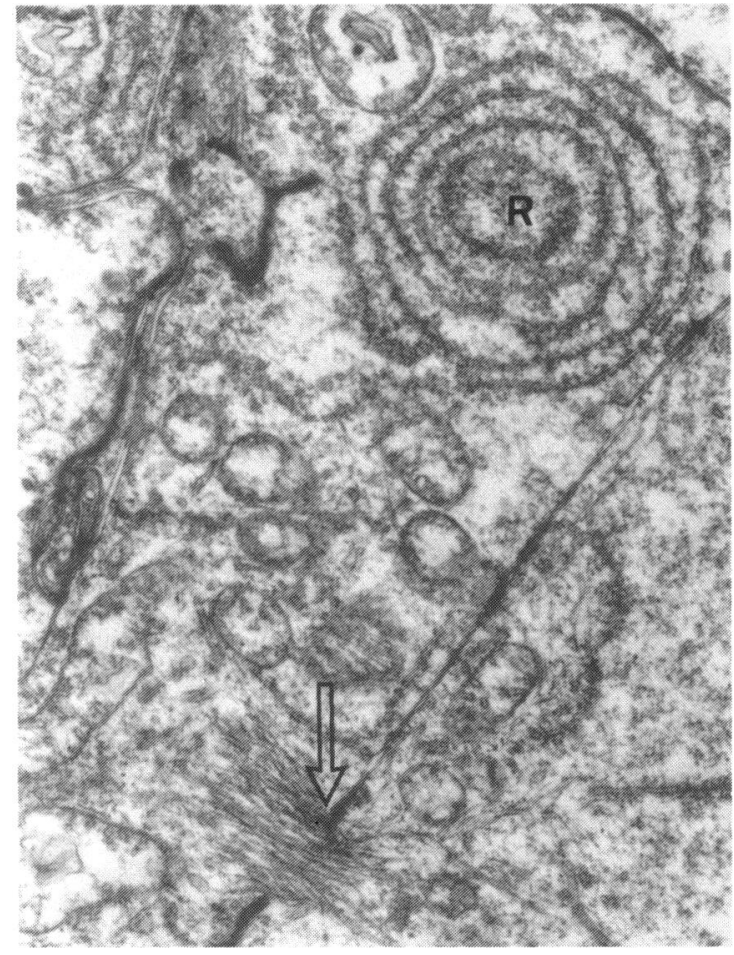

Fig. 8 Intercellular gap (arrow) bridged by intermediate filaments. Note whorl formation of rough endoplasmic reticulum $(\mathrm{R}) . \times 18600)$.

from their substructure, as suggested by Jakobiec. ${ }^{9}$ Nuclear chromatin, endoplasmic reticulum, and other cytoplasmic organelles were not conclusive in distinguishing a 'benign' naevus cell from a 'malignant' melanoma cell. However, the growth pattern in association with the cytological characteristics, enabled us reliably to establish the diagnosis. One year after the exenteration the patient was well and no metastases had been found.

We thank Mrs Leisin and Mrs Mattes for their technical assistance.

\section{References}

1 Rottino A, Kelly A. Primary orbital melanoma. Arch Ophthalmol 1942; 27: 939-49.

2 Jay B. Malignant melanoma of the orbit in a case of oculodermal melanosis (naevus of Ota). Br J Ophthalmol 1965; 49: 359-63.

3 Hagler W, Brown NC. Malignant melanoma of the orbit arising in a nevus of Ota. Ophthalmology 1966; 70: 817-22.

4 Wolter JR, Bryson JM, Blackhurst RT. Primary orbital melanoma. Ear Nose Throat J 1967; 45: 64-7.

5 Jakobiec FA, Ellsworth R, Tannenbaum M. Primary orbital melanoma. Am J Ophthalmol 1974; 78: 24-39.

6 Drews R. Primary malignant melanoma of the orbit in a Negro. Arch Ophthalmol 1975; 93: 335-6.

7 Coppeto JR, Jaffe R, Gillies CG. Primary orbital melanoma. Arch Ophthalmol 1978; 96: 2255-8.

8 Dutton JJ, Anderson RL, Schelper RL, Purcell JJ, Tse DT. 
Orbital malignant melanoma and oculodermal melanocytosis: report of two cases and review of the literature. Ophthalmology 1984: 91: 497-550.

9 Jakobiec FA. The ultrastructure of conjunctival melanocytic tumors. Trans Am Ophthalmol Soc 1984; 82: 599-752.

10 Khalik MK, Duguid WP. Neurotropic malignant melanoma of right temple with orbital metastasis: a clinicopathological case report. Br J Ophthalmol 1987: 71: 41-6.

11 Shields JA, Elder D, Arbizo V, Hedges Th, Augsburger JJ. Orbital involvement with desmoplastic melanoma. $\mathrm{Br} \mathrm{J}$ Ophthalmol 1987: 71: 279-84.
12 Merkow LR, Burt RC, Haysli PDW, Newton FJ, Slifkin M. Pardo M. A cellular and malignant blue nevus. A light and electron microscopic study. Cancer 1969: 24: 888-95.

13 Silverberg GD, Kadin ME. Dorman RL. Haubery JW, Prolo DJ. Invasion of the brain by a cellular blue nevus of the scalp. A case report with light and electron microscopic studies. Cancer 1971: 27: 349-55.

Accepted for publication 14 July 1988. 